\title{
Quantitative anomalous powder diffraction analysis of cation disorder in kesterite semiconductors
}

\author{
Daniel M. Többens ${ }^{1}$, Rene Gunder ${ }^{1,2}$, Galina Gurieva ${ }^{1}$, Julien Marquardt ${ }^{1,2}$, Kai Neldner ${ }^{1,2}$, Laura E. Valle- \\ Rios $^{2}$, Stefan Zander ${ }^{1}$, Susan Schorr ${ }^{1,2}$ \\ ${ }^{1}$ Helmholtz-Zentrum Berlin für Materialien und Energie, Department Structure and Dynamics of Energy \\ Materials
}

${ }^{2}$ Freie Universität Berlin, Institute of Geological Sciences, Malteserstr. 74-100, 12246 Berlin

\begin{abstract}
Kesterite type compound semiconductors, containing copper and zinc, have photovoltaic properties depending on cation distribution in the crystal structure. Anomalous diffraction allows discrimination of isoelectronic cations, in principle allowing a straightforward determination of site occupation factors from data collected at multiple energies close to the X-ray absorption edges of copper and zinc. However, extremely strong correlation between structural parameters precludes this. We present a recipe based on the direct dependency between refined occupation factors and atomic scattering power, which allows to lift the correlations and to detect issues of individual diffraction patterns or assumptions in the model, thereby allowing for reliable quantitative analysis of the $\mathrm{Cu} / \mathrm{Zn}$ distribution.
\end{abstract}

\section{Introduction}

$\mathrm{Cu}_{2} \mathrm{ZnSnS}_{4}$ (CZTS) has received increased attention in recent years. Its properties, namely a nearly ideal band gap of about $1.5 \mathrm{eV}$ and a high absorption coefficient for visible light [1], make it a promising alternative for absorber layers in thin film solar cells. In contrast to other candidates, this compound contains only earth abundant and non-toxic elements. Yielding part of this advantage, properties can be further optimized by varying the composition. In particular, $\mathrm{Cu}_{2} \mathrm{ZnSn}(\mathrm{Se}, \mathrm{S})_{4}$ compounds have already achieved efficiencies above 12\% (Barkhouse, Gunawan, Gokmen, Todorov, \& Mitzi, 2012; Wang et al., 2014). By varying the fractions of copper, zinc, and tin, vacancies and anti-sites can be induced. Moreover, these point defects can likely be controlled by the synthesis conditions (Scragg, Choubrac, Lafond, Ericson, \& Platzer-Bjorkman, 2014). 
Despite large numbers of recent publications dealing with CZTS-derived compounds and the apparent importance of the cation distribution for the photovoltaic properties, knowledge about this is still limited. To a large degree this is due to the problems in distinguishing copper and zinc, which of course is necessary in establishing their distribution. $\mathrm{Cu}^{1+}$ and $\mathrm{Zn}^{2+}$ are isoelectronic cations. As a result, their X-ray scattering characteristics, expressed by the atomic form factor $f$, are nearly identical under conventional circumstances. Standard X-ray diffraction thus cannot distinguish the two elements and can at best provide the amount of vacancies. Methods like XAFS (Bacewicz, Antonowicz, Podsiadlo, \& Schorr, 2014) or Raman spectroscopy (Scragg et al., 2014) have been used to analyze the defect structure. However, direct quantification of cation distribution up to now has only been done successfully from neutron powder diffraction (Schorr, 2011; Schorr, Hoebler, \& Tovar, 2007). The neutron scattering lengths of copper and zinc are sufficiently different from each other, which allows distinction of the two species. Among other things it was settled in these papers that both CZTS and CZTSe compounds crystallize in the kesterite structure, in space group $I \overline{4}$. Application of neutron diffraction is limited by the large amounts of sample typically required, which precludes its application to thin film samples. Also, long data collection times and the dwindling number of neutron sources worldwide limit this method to "corner stone" problems.

Anomalous diffraction in principle can overcome both problems. There are more of the synchrotron sources needed for this method than there are neutron sources, and the high intensity allows using very small sample volumes. As the atomic form factor changes at the absorption edges of the respective elements, $\mathrm{Cu}^{1+}$ and $\mathrm{Zn}^{2+}$ can be distinguished from diffraction data taken with radiation of appropriate energies. Despite this, up to now (A Lafond et al., 2014; Nozaki et al., 2012; Washio et al., 2011) the method has been used only for qualitative analysis, in particular the confirmation of the kesterite structure instead of stannite structure. When quantification of the site occupancies was attempted, this turned out to be unexpectedly problematic. Ideally, it should be possible to simultaneously refine multiple datasets with different wavelengths, and from this to directly derive the site occupancy factors for copper and zinc. In practice, the results of this procedure were found to be unstable. The very high pseudosymmetry of the kesterite structure results in strong correlation between the occupancy factors of the different crystallographic sites. The use of powder samples intensifies this, as symmetry-breaking reflections overlap perfectly. In here, we demonstrate a recipe, by which stable and reliable results can be derived from powder diffraction patterns. 


\section{Theory}

\section{A. Correlation of structural parameters}

Attempts to refine the occupation factors of copper and zinc simultaneously for the same site, using multiple powder diffraction patterns taken close to the respective absorption edges, failed. The results were found to be very unreliable, with extremely high dependency on the values of other parameters. The least-squares correlation matrix yielded correlation factors up to $99 \%$ and higher for actually independent parameters. This can be understood from the particular structural conformation (figure 1). The kesterite structure in space group $I \overline{4}$ derives by cation ordering from the sphalerite structure in space group $F \overline{4} 3 m$. As a result the geometry of the four cation sites $2 a-2 d$ is very similar. Changes to the structure factor due to changes in the occupation are consequently similar and can be balanced to a large degree by changes in another site. This effect is particularly strong for the pair $2 c-2 d$, which only split at the transition from disordered $(I \overline{4} 2 m)$ to ordered $(I \overline{4})$ kesterite. Similarily, the coordinates of the anion, $x y z$ with $x \approx y$, result in strong correlations between $x$ and $y$ and anion displacement parameters.

In powder diffraction the situation is complicated further by the fact that the symmetry of Kesterite is merohedral. As $h k l$ and $k h l$ reflections are perfectly superimposed, it becomes impossible for the refinement algorithm to distinguish $x$ from $y$ and $2 c$ from $2 d$. This also holds in single crystals, as twinning by merohedry is virtually unavoidable in this situation. In a number of cases during the course of preparing this paper this led to the occupation of $2 c$ and $2 d$ by copper and zinc coming out exchanged, with $\mathrm{Zn}$ preferably on $2 c$ and $\mathrm{Cu}$ on $2 d$. Such an apparent exchange of $2 c$ and $2 d$ is structurally equivalent to an exchange of the very barely discriminable anion coordinates $x$ and $y$.

In such a situation, a single bad data set or a parameter fixed to an incorrect value can result in refined values, which deviate strongly from the true value, without an obvious reduction in fit quality. It is thus necessary to employ a method of analysis that allows detection of such errors.

\section{B. Linearization}

When refining an occupation factor $o c c_{j}^{c a l c}$ of a crystallographic site $j$, the quantity actually refined is the average scattering power of the atoms located at the crystallographic site. For the interaction with Xrays, the scattering power of an atom is described by the atomic form factor $f=f_{0}+f^{\prime}+\mathrm{i} \cdot f^{\prime}$. The total scattering power $t s p_{j}$ of a crystallographic site is the product of atomic form factor and site occupation 
factor, occ.f. In case the site is occupied by multiple chemical species, the total scattering power is the sum of the contribution of all species

$$
t s p=\sum_{X} o c c(\mathrm{X}) \cdot f(X)=\sum_{X} o c c(\mathrm{X}) \cdot\left(f_{0}(\mathrm{X})+f^{\prime}(\mathrm{X})+i \cdot f^{\prime \prime}(\mathrm{X})\right)
$$

If the chemical occupation of the site is unknown, the real total scattering power can be refined in good approximation from a model containing only one element $A$, which might or might not be part of the actual mix:

$$
\operatorname{tsp}(\text { model })=\operatorname{occ}^{c a l c}(\mathrm{~A}) \cdot f(\mathrm{~A})=\operatorname{occ}^{\text {calc }}(\mathrm{A}) \cdot\left(f_{0}(\mathrm{~A})+f^{\prime}(\mathrm{A})+i \cdot f^{\prime \prime}(\mathrm{A})\right)
$$

Here and in the following occ calc is used when referring to site occupation factors of the model, while occ is used for the real site occupation factors in the crystal structure. In the absence of any other sources of error, a refinement will yield

$$
\begin{aligned}
& t s p(\text { model })=t s p \\
& \operatorname{occ}^{\operatorname{calc}}(\mathrm{A}) \cdot f(\mathrm{~A})=\sum_{X} \operatorname{occ}(\mathrm{X}) \cdot f(\mathrm{X})
\end{aligned}
$$

If one is interested in the occupation occ of a particular species B we can split its contribution from the sum at the right side of the equation, as well as the contribution of species $A$

$$
\operatorname{occ}^{\operatorname{calc}}(A) \cdot f(\mathrm{~A})=\operatorname{occ}(\mathrm{B}) \cdot f(B)+\operatorname{occ}(\mathrm{A}) \cdot f(A)+\sum_{X \neq A, B} \operatorname{occ}(\mathrm{X}) \cdot f(\mathrm{X})
$$

that can be rewritten as

$$
\operatorname{occ}^{\operatorname{calc}}(\mathrm{A})=\operatorname{occ}(\mathrm{B}) \cdot \frac{f(\mathrm{~B})}{f(\mathrm{~A})}+\operatorname{occ}(\mathrm{A})+\frac{1}{f(\mathrm{~A})} \sum_{\mathrm{X} \neq \mathrm{A}, \mathrm{B}} \operatorname{occ}(\mathrm{X}) \cdot f(\mathrm{X})
$$

In conventional X-ray or neutron diffraction, information about the chemical composition of the sample is necessary to determine the element distribution from this relation (Schorr, 2011; Schorr et al., 2007). Anomalous X-ray scattering, however, exploits the fact that $f^{\prime}$ and $f^{\prime \prime}$ are wavelength dependent (figure 2). It is thus possible to use $f(B) / f(A)$ as a variable against which $\operatorname{occ}^{\text {calc }}(A)$ can be plotted. A proper definition of this variable is not trivial, as the atomic form factor $f=f_{0}(2 \theta)+f^{\prime}(\lambda)+i \cdot f^{\prime}(\lambda)$ is rather complicated: While the dispersion factor $f^{\prime}$ is a constant for a given X-ray wavelength, the base component $f_{0}$ is a function of the diffraction angle. Finally, the phase shift described by $f^{\prime \prime}$ turns the form 
factor into a complex function; how this affects the intensities depends on both the site of the atom in the structure and on the Miller indices of the reflection. However, we will show that the simple approximation $f \approx\left\langle f_{0}\right\rangle+f^{\prime}$ is sufficient to describe the system, if a properly adjusted value for $\left\langle f_{0}\right\rangle$ is chosen. While it is not clear how the average $\left\langle f_{0}>\right.$ of $f_{0}(2 \theta)$ would have to be calculated theoretically, it can easily be derived numerically from simulated or experimental diffraction patterns of fully ordered structures.

With this approximation, the abovementioned equation gives rise to useful applications. In the simple case of only two elemental species, A and B, it becomes

$$
\operatorname{occ}^{\operatorname{calc}}(\mathrm{A})=\operatorname{occ}(\mathrm{B}) \cdot \frac{f(\mathrm{~B})}{f(\mathrm{~A})}+\operatorname{occ}(\mathrm{A})
$$

Thus a plot of $\operatorname{occ}^{c a l c}(\mathrm{~A})$ over $f(\mathrm{~B}) / f(\mathrm{~A})$ is linear (figure 3), with $\operatorname{occ}(\mathrm{B})$ as slope, $\operatorname{occ}(\mathrm{A})$ as zero intercept and the total site occupation $\operatorname{occ}(\mathrm{A})+\operatorname{occ}(\mathrm{B})$ at $f(\mathrm{~B}) / f(\mathrm{~A})=1$. It has to be pointed out that measurements at the absorption edges of both elements $A$ and $B$ can be described with the same line. As $f^{\prime}$ close to the edge will become strongly negative, $f \approx\left\langle f_{0}\right\rangle+f^{\prime}$ will decrease for this element, while it remains constant in first approximation for the other element. Thus the data at the two absorbtion edges will form two arms of the line, with the values at the A-edge at $f(\mathrm{~B}) / f(\mathrm{~A})>1$ and those at the B-edge at $f(\mathrm{~B}) / f(\mathrm{~A})<1$.

If additional elemental species are present at the particular site, systematic deviations from linearity will occur, expressed by the additional contribution

$$
\frac{1}{f(\mathrm{~A})} \sum_{X \neq A, B} \operatorname{occ}(\mathrm{X}) \cdot f(\mathrm{X})
$$

The effects of this summand on $\operatorname{occ}(\mathrm{A})$ are different for the $\mathrm{A}$ - and $\mathrm{B}$-arms (figure 4). In the latter, $f(\mathrm{~A})$ is nearly constant. $f(B)$ is not, but does not affect the additional summand. As long as no element $X \neq A, B$ is present, which happens to have an edge in the observed energy range, all the components of the summand are constant, too. Therefore, the refined occupation value $\operatorname{occ}(A)$ will be increased by a constant value for the data points of the B-arm.

In the A-arm the situation is different, as $f(\mathrm{~A})$ is reduced close to the absorption edge of element $\mathrm{A}$, while $f(B)$ is fairly constant. As a result the deviation from the ideal line formed by both arms is proportional to the abscissa $f(\mathrm{~B}) / f(\mathrm{~A})$. In addition to the constant increase in the B-arm, the A-arm will show an increased slope; the line will appear bent at $f(B) / f(A)=1$. 
The amounts of $A, B$, and other elements $X$ on a particular site can be calculated from the differences between the two arms in a straightforward manner. In the B-arm, the slope is occ(B), while the intercept is $\operatorname{occ}(\mathrm{A})+\operatorname{occ}(\mathrm{X}) \cdot f(\mathrm{X}) / f(\mathrm{~A})$. In the $\mathrm{A}$-arm, the slope is $\operatorname{occ}(\mathrm{B})+\operatorname{occ}(\mathrm{X}) \cdot f(\mathrm{X}) / f(\mathrm{~B})$, while the intercept is $\operatorname{occ}(\mathrm{A})$. The difference between the two slopes as well as between the two intercepts yields $\operatorname{occ}(\mathrm{X})$, provided $f(\mathrm{X})$ is known.

\section{Adjustment of parameters}

\section{A. Scattering power ratio and slope}

The correct numerical value to be used for $f_{0}$ can be derived from simulations, in which theoretical diffraction patterns are calculated with one species B occupying a site and subsequently analyzed with another species $\mathrm{A}$ in the model. A plot of the results for different values of $f^{\prime}(\mathrm{A})$ and $f^{\prime}(\mathrm{B})$ will result in a line with slope and intercept depending on $f_{0}(\mathrm{~A})$ and $f_{0}(\mathrm{~B})$, with slope $=1$ and intercept $=0$ for the appropriate values. Figure 3 shows this for copper and zinc in kesterites. The structural data of a fully ordered CZTS single crystal (L. Choubrac, Lafond, Guillot-Deudon, Moëlo, \& Jobic, 2012) and dispersion correction factors of Cromer and Liberman (Cromer \& Liberman, 1981) were used to calculate powder diffraction data for multiple energies. In this model, the cations were completely ordered, with copper occupying $2 a$ and $2 c$, and zinc occupying $2 d$. It is trivial that a Rietveld refinement of the simulated data using the same model yields site occupation factors of 1.0. In subsequent analysis, occupation with copper and zinc was exchanged for a single site in the model, assuming $\mathrm{Zn} 2 a, \mathrm{Zn} 2 c$, or $\mathrm{Cu} 2 d$, respectively. This results in refined site occupation factors occ ${ }^{\text {calc }}$ different from 1.0. As discussed above, plotting these values against scattering power ratios, $f(\mathrm{Zn}) / f(\mathrm{Cu})$ for sites assumed as $\mathrm{Cu}$-occupied in the analysis and $f(\mathrm{Cu}) / f(\mathrm{Zn})$ for sites assumed as $\mathrm{Zn}$-occupied, results in linear functions (figure 3 ). The appropriate values for $\left\langle f_{0}>\right.$ are those resulting in a slope $=1$.

Ideal powder diffraction data, with FWHM $\rightarrow 0$ and $2 \theta$-range of $0^{\circ}$ through $180^{\circ}$, give

$$
f(\mathrm{Cu})=20.4+f^{\prime}(\mathrm{Cu}) \text { and } f(\mathrm{Zn})=21.3+f^{\prime}(\mathrm{Zn}) .
$$

There are some changes in refined $o c c^{\text {calc }}$ with both the high-angle limit and the peak shape and width in the diffraction pattern.

Between the $180^{\circ}$ ideal limit and the $105^{\circ}$ limit used in our experimental tests, the latter has about $8 \%$ lower slope. A realistic peak width and shape increases this effect; typically high-angle peaks are broader 
and influence the Rietveld fit less than the sharp and intense peaks at low angle. Using the peak shape from our experiments for the simulations, this effect is once again of about $8 \%$ strength.

For simulations using the peak width and high angle limit corresponding to our experimental setup $\left(<\mathrm{FWHM}>\approx 0.1^{\circ}, 2 \theta>105^{\circ}\right)$, the appropriate values were

$$
f(\mathrm{Cu})=23.2+f^{\prime}(\mathrm{Cu}) \text { and } f(\mathrm{Zn})=24.0+f^{\prime}(\mathrm{Zn}) .
$$

With these values correct slopes and intercepts were derived from both Cu-modelled sites plotted as function of $f(\mathrm{Zn}) / f(\mathrm{Cu})$ and of $\mathrm{Zn}$-modelled sites plotted over $f(\mathrm{Cu}) / f(\mathrm{Zn})$.

Both linearity and the values needed for the adjusted scattering factor ratio are mostly independent of the chemical type of the anion in the compound. In particular, in selenides the scattering power of the anion is doubled compared to sulfides. However, the resulting slope of the lines did not change significantly; it was decreased by $0.016(7)$. The same holds for the intercepts, which increased correspondingly. For any realistic level of experimental accuracy this has to be considered insignificant.

The influence of the $f^{\prime \prime}$ component of the scattering factor has been neglected in this analysis. As an imaginary component, the effect of $f^{\prime \prime}$ on the scattering power of a particular site is not the simple direct proportionality of the other components. In particular, the effect of $f^{\prime \prime}$ on the structure factor depends on the coordinates of the site in the crystal structure. It is thus not possible to give one equation that is valid for all cation sites in the structure. Thus, considering $f^{\prime \prime}$ in the analysis leads to a much more complicated recipe. Fortunately, $f^{\prime \prime}$ is comparatively small for copper and zinc; its change at the absorption edges is about 3 electrons. Nonetheless, its effect can be seen (figure 3): A small discontinuity

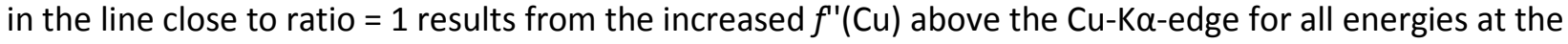
$\mathrm{Zn}-\mathrm{K} \alpha$-edge. More pronounced is the offset in the data points at the extremes of both arms, which results from energies just above the absorption edge, where the respective $f^{\prime \prime}$ is at a maximum. However, it is trivial to avoid these energies in the experimental data collection. Since above the absorption edge the background is very high due to incoherent scattering, they are inferior in any case. Also the slightly different slopes of the lines for the three different cation sites are a result of the different $f^{\prime \prime}$ values of copper and zinc. If these are set to zero in the simulation, the divergence vanishes together with perfect linearity appearing. 
As discussed above, occupation of a site by an element different from the two considered in the ratio results in distinct deviations from linearity. The data plotted in figure 4 result from models of the kesterite structure, in which one additional cation site was assumed to be fully occupied by tin (Sn). The diffraction data simulated from these models were subsequently analyzed in the previously described way, assuming either copper or zinc on the respective site. In the right arms, at the absorption edge of the element assumed in the model, a slope of 1.81(8) is found for assumed $\mathrm{Cu}$, and of 1.82(12) for assumed $\mathrm{Zn}$. Both lines have intercepts not significantly different from zero. In the left arm, the absolute refined occupation factor at the extreme ratios, far away from ratio $=1$, is $1.85(2)$ for $\mathrm{Cu}$ and $1.83(3)$ for $\mathrm{Zn}$. All parts of the evaluation thus result in the same value for the occupation factor, which is too high by a factor of 1.83. Accordingly, $f(\mathrm{X}) / f(\mathrm{Cu})=f(\mathrm{X}) / f(\mathrm{Zn})=1.83$ can be used to calculate the site occupation factor of $\mathrm{X}=\mathrm{Sn}$ as explained in the theory section. This ratio is expected if the scattering factor $f(\mathrm{Sn})=$ $f_{0}(\mathrm{Sn})+f^{\prime}(\mathrm{Sn})$ is calculated using $f_{0}(\mathrm{Sn})=44$, which is lower than the number of electrons in the element by approximately the same value of 6 as was used for copper and zinc. Obviously, this cannot be valid with the same constant for all elements; in particular vacancies can be treated as a special case, in which both the number of electrons and the scattering factor are zero.

There are some considerable deviations from the idealized behavior. Most pronounced is the relative reduction in $\operatorname{occ}^{\text {calc }}(\mathrm{Zn})$ in the data points closest to ratio $=1$. The values were derived using an energy of $12398 \mathrm{eV}$, well above the absorption edges of both copper and zinc. These deviations result from the rather high $f^{\prime \prime}(\mathrm{Sn})$, not considered in the analysis. This results from the L1-absorption edge of tin at 4465 $\mathrm{eV}$; over the energy range considered in this analysis, $f^{\prime}(\mathrm{Sn})$ falls from 5.5 to 2.6 with increasing energy. Ironically, it is only above the absorption edges of both copper and zinc that $f^{\prime \prime}$ is roughly equal for $\mathrm{Sn}$, $\mathrm{Cu}$, and $\mathrm{Zn}$. These data points thus should be more correct. However, as the general analysis calls for values below the edges, it is prudent to adjust numerical factors accordingly.

\section{B. Experimental values for scattering factors}

For all components of the atomic form factor, various tabulated values exist. In this work we used for the wavelength-independent part $f_{0}$ the analytic 9-parameter approximation stored in Fullprof (RodríguezCarvajal, 2012). The parameters for neutral atoms were used, which were found to work slightly, but not significantly, better than those for cations. The anomalous factors $f^{\prime}$ and $f^{\prime \prime}$ were taken from the X-ray Anomalous Scattering website of E. A. Merritt (Merritt, 2014). These scattering factor data were calculated using the subroutine library by Brennan and Cowan (Brennan \& Cowan, 1992), in turn using 
the theoretical approximation developed by Cromer and Liberman (Cromer \& Liberman, 1981). Values between tabulated energies were derived by linear interpolation.

Cromer and Liberman theory gives accurate values far from an absorption edge but does not account for the effects of neighboring atoms, which can be very substantial near an absorption edge. For the purpose of the analysis detailed in here, this effect is best gauged from a site, which is fully occupied only by the one chemical species whose form factor parameters are tested. In this case and in the absence of any other sources of error the occupation factors refined using the scattering factor for the correct element should all give the correct $o c c^{c a l c}=o c c=1$. The refined value might deviate from 1 if either the site occupation factor is less than 1 or if correlated parameters, e.g. the thermal displacement parameters, are incorrect. But even in these cases the refined values should be constant. The crystal structure of the compound used for this calibration has to be similar to the structure of the real samples for values to be transferable.

A sufficient number of different energies both close to the edge and far away from it should be measured to allow distinction between different sources of error: 1) Estimated standard deviations from Rietveld refinements tend to be too small (Bérar \& Lelann, 1991); the real uncertainty of the results is higher. For those values far from the absorption edge, this is the factor determining the data scattering. 2) If the anomalous scattering factors close to the absorption edge are significantly different from theory, the refined occ at these energies deviate from the constant more than average. As the deviation from theory is probably systematic, the horizontal line can be expected to gain a hockey stick bend. 3 ) The relative position of the absorption edge might be shifted. This can happen from a real shift of the absorption edge compared to the values for the pure element (Kumar, Nagarajan, \& Sarangi, 2013). It might also be the result of an error in the energy calibration of the experiment. 4) If the assumption of a fully ordered structure is not correct and some amount of other chemical elements occupies the site, the deviations from linearity described in the previous section will be observed.

It should be obvious that the high number of potential systematic errors makes it necessary to measure a rather large number of different energies both close to the edge and farther away from it.

For the examination of the $f^{\prime}$-values of copper a stoichiometric CZTS sample cooled down at a rate of 1 $\mathrm{K} / \mathrm{h}$ was used. Neutron diffraction analysis (Schorr et al., 2007) showed that in this sample the $2 a$ position is fully occupied by copper only. All occ calc data points far away from the Cu-K edge are constant within \pm 0.01 (figure 5). The average occupation calculated from these values is slightly increased, with $\left\langle\right.$ occ $\left.^{\text {calc }}\right\rangle=1.021(7)$. As discussed above, this might have any number of reasons and it is not justified to $9 / 21$ 
use $f^{\prime}$ to adjust this. In contrast, the two refined occupancies close to the Cu-K edge are significantly increased. This indicates too low scattering power attributed to copper in the model, thus $f^{\prime}$ too negative. To bring these values down to the average, the values of $f$ had to be changed, at $8969 \mathrm{eV}$ from -6.5604 to -6.26 , at $8974 \mathrm{eV}$ from -7.4207 to -6.70 (Table I). Experimental $f$ at the Cu-K edge is less pronounced than predicted by theory. This is expected, as real structure effects will tend to broaden the sharp theoretical curve.

For the scattering factors of $\mathrm{Zn}$ a sample of pure sphalerite, $\mathrm{ZnS}$, was analyzed. Sphalerite has strong cleavability along $\{110\}$ (Kleeberg \& Mibus, 2010), which was considered in the Rietveld analysis by a preferred orientation model using the modified March function. The resulting occupation factors of zinc are slightly below 1 , with $\left\langle\operatorname{occ}^{\text {calc }}\right\rangle=0.993(9)$. Significant deviation from the theoretical value of $f$ was observed at the energy closest to the edge; $f^{\prime}$ at $9654 \mathrm{eV}$ was changed from -7.5581 to -7.20.

\section{Examples of practical application}

The method described was applied to a well characterized CZTSSe sample, a monograin powder previously analyzed by neutron powder diffraction. Cation ratios $\mathrm{Cu} /(\mathrm{Zn}+\mathrm{Sn})=0.944$ and $\mathrm{Zn} / \mathrm{Sn}=1.039$ of the kesterite type phase were determined by wavelength dispersive X-ray spectroscopy (WDX). This corresponds to an A-type kesterite (A. Lafond, Choubrac, Guillot-Deudon, Deniard, \& Jobic, 2012) and a chemical composition as $\mathrm{Cu}_{1.942} \mathrm{Zn}_{1.049} \mathrm{Sn}_{1.009} \mathrm{~S}_{3.288} \mathrm{Se}_{0.712}$.. The sample contains small amounts of two secondary phases, determined by WDX and PXRD, 5.1(1.8)\% ZnS-type phase $\mathrm{Zn}_{0.95} \mathrm{Cu}_{0.04} \mathrm{Sn}_{0.01} \mathrm{~S}_{0.9} \mathrm{Se}_{0.1}$ and 2.8(5)\% $\mathrm{Cu}_{2} \mathrm{SnS}_{3}$-type phase $\mathrm{Cu}_{1.5} \mathrm{Zn}_{0.5} \mathrm{SnS}_{2.3} \mathrm{Se}_{0.7}$. Visible peaks of the latter were excluded from the refinement. The peaks of the ZnS-type phase overlap with the main peaks of the kesterite phase. Considering this phase resulted in negligible changes in the results. The procedure applied in this particular case is intended as an example only; it should be adapted to the particular experiment:

1) Data collection: Anomalous X-ray powder diffraction data (AXPD) were collected the diffraction end station of the KMC-2 beamline (Erko et al., 2000; Helmholtz-Zentrum Berlin für Materialien und Energie, 2016) at BESSY II (Berlin, Germany). KMC-2 provides X-ray radiation with both very stable energies and intensities. The accessible energy range of $4-14 \mathrm{keV}$ is ideally suited for the K-edges of $\mathrm{Cu}(8979 \mathrm{eV})$ and Zn $(9659 \mathrm{eV})$. In the experiments described here eight different energies were used, one far below the 
absorption edge (8048 eV), three each at 60, 10, and $5 \mathrm{eV}$ below the absorption edges of copper and zinc, and one above (12398 eV). Total data collection time for one sample was 5 hours, for a $2 \theta$-range of $5-104^{\circ}$ and about 60,000 counts in the strongest peak at an integration step size of $0.01^{\circ}$. The sample was in symmetric reflection geometry at ambient condition. Sufficient particle statistic was ensured by rotating the sample around an excentric point outside of the illuminated area, and by using a high number of $\theta-2 \theta$-positions in combination with an area detector (Vantec 2000) with $12^{\circ}$ acceptance angle both vertically and horizontally. As in this geometry the symmetric reflection condition is not strictly conserved, an appropriate absorption correction was applied during data refinement (Wronski et al., 2009).

2) LeBail refinements were done using Fullprof.2k (Rodríguez-Carvajal, 2001) to determine the optimum fit profile model. In this case a Pearson VII peak shape was adopted. Systematic peak shifts and anisotropic peak broadening were tested, but found to be insignificant. Background was interpolated between positions with no or little intensity contribution from peaks. Regions containing small peaks from the impurity were excluded from the refinement. Peak width, asymmetry and background were refined and fixed for subsequent Rietveld analysis.

3) The structure model was taken from the ordered CZTS kesterite ICSD 262388 (L Choubrac, Lafond, Guillot-Deudon, Moelo, \& Jobic, 2012), which was refined from single-crystal data. The anisotropic displacement parameters of all atoms were retained. The anion occupancy was modified to reflect the composition of the sample; differences resulting from nominal $(S=3.25$, Se $=0.75)$ or microprobe $(S=$ 3.288 , Se $=0.712$ ) composition were found insignificant. Cation occupation of the sites were assigned as $\mathrm{Cu} 2 a, \mathrm{Cu} 2 c, \mathrm{Zn} 2 d, \mathrm{Sn} 2 b$, each fully occupied, that is the ideal ordered kesterite type structure. In this stage only the $8048 \mathrm{eV}$ data set was used, where the unknown Cu/Zn distribution has little influence. The data set at $12398 \mathrm{eV}$ gave results with higher uncertainties due to the increased background from copper and zinc fluorescence. The use of an energy-discriminating detector might be useful here. Scale factor and lattice were set as refineable parameters. Structural parameters refined in the preparatory stage were S,Se coordinates $x y z$ and an overall displacement parameter. The occupancy of Sn $2 b$ was tested, but did not differ significantly from full occupation. The same holds for preferred orientation. The refined parameters were subsequently transferred to all data sets and fixed for subsequent analysis.

4) Occupation factors of $\mathrm{Cu} 2 a, \mathrm{Cu} 2 c, \mathrm{Zn} 2 d$ were independently refined for all eight data sets.

5) Subsequent data analysis was done using Origin 9.0. The results from step 4 were plotted (figure 6) as function of $f(\mathrm{Zn}) / f(\mathrm{Cu})$ or $f(\mathrm{Cu}) / f(\mathrm{Zn})$, using the scattering factors $f(\mathrm{Cu})=23.2+f^{\prime}(\mathrm{Cu})$ and $f(\mathrm{Zn})=24.0+$ $11 / 21$ 
$f^{\prime}(\mathrm{Zn})$, as determined from simulations. The data were checked for linearity within statistical uncertainty and for impossible trends, in particular negative slopes, whose presence would have indicated problems in either experiment or analysis procedure. Regression lines for the three sites were fitted by least squares procedure, with the data points weighted by their Rietveld-derived e.s.d.s.

6) The results are given in table II, together with occupancies derived from a previous analysis by neutron diffraction. It might be necessary to normalize the results to comply with total composition from chemical analysis or charge balance. This was not necessary in this case. In a A-type kesterite, expected defects due to deviation from stoichiometry are copper vacancies $\left(\mathrm{V}_{\mathrm{Cu}}\right)$ and $\mathrm{Zn}_{\mathrm{Cu}}$, where extra zinc is located at Cu-sites. Both are generally concentrated at the $2 a$ site (A. Lafond et al., 2012). The latter are confirmed in this AXRPD analysis. The analysis gives no indication of vacancies; however, their expected number is below uncertainties. Additional $\mathrm{Cu} / \mathrm{Zn}$ disorder on the $2 c$ and $2 d$ sites is found correctly.

\section{Conclusion}

Anomalous diffraction at the absorption edges of copper and zinc allows quantitative determination of the cation disorder in CZTSSe materials. The attainable accuracy is at the same level as can be reached by neutron diffraction. Due to the particular crystal structure of kesterites, strong correlations between structural parameters are unavoidable. Extremely careful experimentation and analysis is needed to avoid and detect systematic errors. Even under the best conditions, some uncertainty about the accuracy of the obtained absolute values of site occupation factors remains. Combining the raw results with additional information, e.g. chemical analysis, should allow resolving this. The same holds for parametric studies, in which multiple samples are measured and analyzed in the same way.

\section{Acknowledgement}

Measurements were carried out at the Diffraction instrument at KMC-2 beamline at Helmholtz-Zentrum Berlin für Materialien und Energie. We thank HZB for the allocation of synchrotron radiation beamtime. 


\section{Literature}

Bacewicz, R., Antonowicz, J., Podsiadlo, S., and Schorr, S. (2014). "Local structure in Cu2ZnSnS4 studied by the XAFS method," Solid State Communications, 177, 54-56.

Barkhouse, D., Gunawan, O., Gokmen, T., Todorov, T., and Mitzi, D. (2012). Device characteristics of a 10.1\% hydrazine-processed Cu2ZnSn(Se,S)4 solar cell," Progress in Photovoltaics, 20(1), 6 - 11.

Brennan, S., and Cowan, P. L. (1992). "A suite of programs for calculating x-ray absorption, reflection and diffraction performance for a variety of materials at arbitrary wavelengths," Review of scientific instruments, 63, $850-853$.

Bérar, J.-F., and Lelann, P. (1991). "E.S.D.'s and estimated probable error obtained in Rietveld refinements with local correlations," Journal of Applied Crystallography, 24(1), 1 - 5.

Choubrac, L., Lafond, A., Guillot-Deudon, C., Moëlo, Y., and Jobic, S. (2012). "Structure Flexibility of the Cu2ZnSnS4 Absorber in Low-Cost Photovoltaic Cells: From the Stoichiometric to the Copper-Poor Compounds," Inorganic Chemistry, 51(6), 3346 - 3348.

Cromer, D. T., and Liberman, D. A. (1981). "Anomalous dispersion calculations near to and on the longwavelength side of an absorption edge," Acta Crystallographica A, 37, 267 - 268.

Erko, A., Packe, I., Hellwig, C., Fieber-Erdmann, M., Pawlizki, O., Veldkamp, M., and Brennan, S. (2000). "KMC-2: the new $\mathrm{x}$-ray beamline at BESSY II," Synchrotron Radiation Instrumentation, 521, 415 418.

Hall, S. R., Szymanski, J. T., and Stewart, J. M. (1978). "Kesterite, Cu2(Zn,Fe)SnS4, and stannite, Cu2(Fe,Zn)SnS4, structurally similar but distinct minerals," The Canadian Mineralogist, 16(2), 131 $-137$.

Helmholtz-Zentrum Berlin für Materialien und Energie. (2016). KMC-2: an X-ray beamline with dedicated diffraction and XAS endstations at BESSY II. Journal of large-scale research facilities, 2, A49.

Kleeberg, R., and Mibus, J. (2010). "BGMN Application: Copper Shale (Kupferschiefer)," Retrieved from http://www.bgmn.de/copper.html.

Kumar, P., Nagarajan, R., and Sarangi, R. (2013). "Quantitative X-ray absorption and emission spectroscopies: electronic structure elucidation of Cu2S and CuS," Journal of Materials Chemistry C, 1(13), 2448 - 2454.

Lafond, A., Choubrac, L., Guillot-Deudon, C., Deniard, P., and Jobic, S. (2012). "Crystal Structures of Photovoltaic Chalcogenides, an Intricate Puzzle to Solve: the Cases of CIGSe and CZTS Materials," Zeitschrift Für Anorganische Und Allgemeine Chemie, 638(15), 2571 - 2577.

Lafond, A., Choubrac, L., Guillot-Deudon, C., Fertey, P., Evain, M., and Jobic, S. (2014). "X-ray resonant single-crystal diffraction technique, a powerful tool to investigate the kesterite structure of the photovoltaic Cu2ZnSnS4 compound," Acta Crystallographica Section B-Structural Science Crystal Engineering and Materials, 70, 390 - 394.

Merritt, E. A. (2014). "X-ray Anomalous Scattering," Retrieved from http://skuld.bmsc.washington.edu/scatter/.

Nozaki, H., Fukano, T., Ohta, S., Seno, Y., Katagiri, H., and Jimbo, K. (2012). "Crystal structure determination of solar cell materials: Cu2ZnSnS4 thin films using X-ray anomalous dispersion," Journal of Alloys and Compounds, 524, 22 - 25.

Origin 9.0. OriginLab, Northampton, MA.

Rodríguez-Carvajal, J. (2001). "Recent developments of the program FULLPROF," Commission on powder diffraction (IUCr). Newsletter, 26, 12 - 19.

Rodríguez-Carvajal, J. (2012). Fullprof.2k (Version 5.30). ILL-JRC.

Schorr, S. (2011). "The crystal structure of kesterite type compounds: A neutron and X-ray diffraction study," Solar Energy Materials and Solar Cells, 95(6), 1482 - 1488.

Schorr, S., Hoebler, H., and Tovar, M. (2007). "A neutron diffraction study of the stannite-kesterite solid solution series," European Journal of Mineralogy, 19(1), 65 - 73. 
Scragg, J., Choubrac, L., Lafond, A., Ericson, T., and Platzer-Bjorkman, C. (2014). "A low-temperature order-disorder transition in Cu2ZnSnS4 thin films," Applied Physics Letters, 104(4), 041911.

Wang, W., Winkler, M. T., Gunawan, O., Gokmen, T., Todorov, T. K., Zhu, Y., and Mitzi, D. B. (2014). "Device Characteristics of CZTSSe Thin-Film Solar Cells with 12.6\% Efficiency," Advanced Energy Materials, 4(7), 1301465.

Washio, T., Nozaki, H., Fukano, T., Motohiro, T., Jimbo, K., and Katagiri, H. (2011). "Analysis of lattice site occupancy in kesterite structure of Cu2ZnSnS4 films using synchrotron radiation x-ray diffraction," Journal of Applied Physics, 110(7), 074511.

Wronski, S., Wierzbanowski, K., Baczmanski, A., Lodini, A., Braham, C., and Seiler, W. (2009). "X-ray grazing incidence technique-corrections in residual stress measurement - a review," Powder Diffraction, 24(2), 11 - 15. 


\section{Tables}

Table I. Anomalous scattering factors $f^{\prime}$ of copper and zinc (Cromer and Liberman). Values marked * have been adjusted from the original value given in brackets.

\begin{tabular}{|l|l|l|}
\hline Energy / eV & $\boldsymbol{f}^{\prime}(\mathrm{Cu})$ & $f^{\prime}(\mathrm{Zn})$ \\
\hline 8048 & -1.9635 & -1.5465 \\
\hline 8919 & -4.6172 & -2.2235 \\
\hline 8955 & -5.5884 & -2.2689 \\
\hline 8969 & $-6.26^{*}(-6.5604)$ & -2.2866 \\
\hline 8974 & $-6.70^{*}(-7.4207)$ & -2.2929 \\
\hline 9376 & -2.3273 & -3.1232 \\
\hline 9599 & -1.8036 & -4.6777 \\
\hline 9635 & -1.7422 & -5.6561 \\
\hline 9649 & -1.7185 & -6.6476 \\
\hline 9654 & -1.7100 & $-7.20 *(-7.5581)$ \\
\hline 12398 & -0.0745 & -0.4026 \\
\hline
\end{tabular}

Table II. Results of AXRPD analysis and neutron analysis. Note that neutron results given for comparison are necessarily normalized to the results of the chemical analysis. The $2 b$ site is occupied by tin.

\begin{tabular}{|l|l|l|l|l|l|l|}
\hline & \multicolumn{2}{|l}{ AXRPD } & \multicolumn{2}{l|}{ NPD } \\
\hline & Cu & Zn & Total & Cu & Zn & Total \\
\hline $2 a$ & $0.961(45)$ & $0.048(40)$ & 1.009 & 0.890 & 0.090 & 0.98 \\
\hline $2 c$ & $0.621(53)$ & $0.398(48)$ & 1.019 & 0.600 & 0.400 & 1 \\
\hline $2 d$ & $0.438(50)$ & $0.556(45)$ & 0.994 & 0.450 & 0.550 & 1 \\
\hline Total & 2.020 & 1.002 & & 1.94 & 1.04 & \\
& & & & & & \\
\hline
\end{tabular}




\section{Figures}

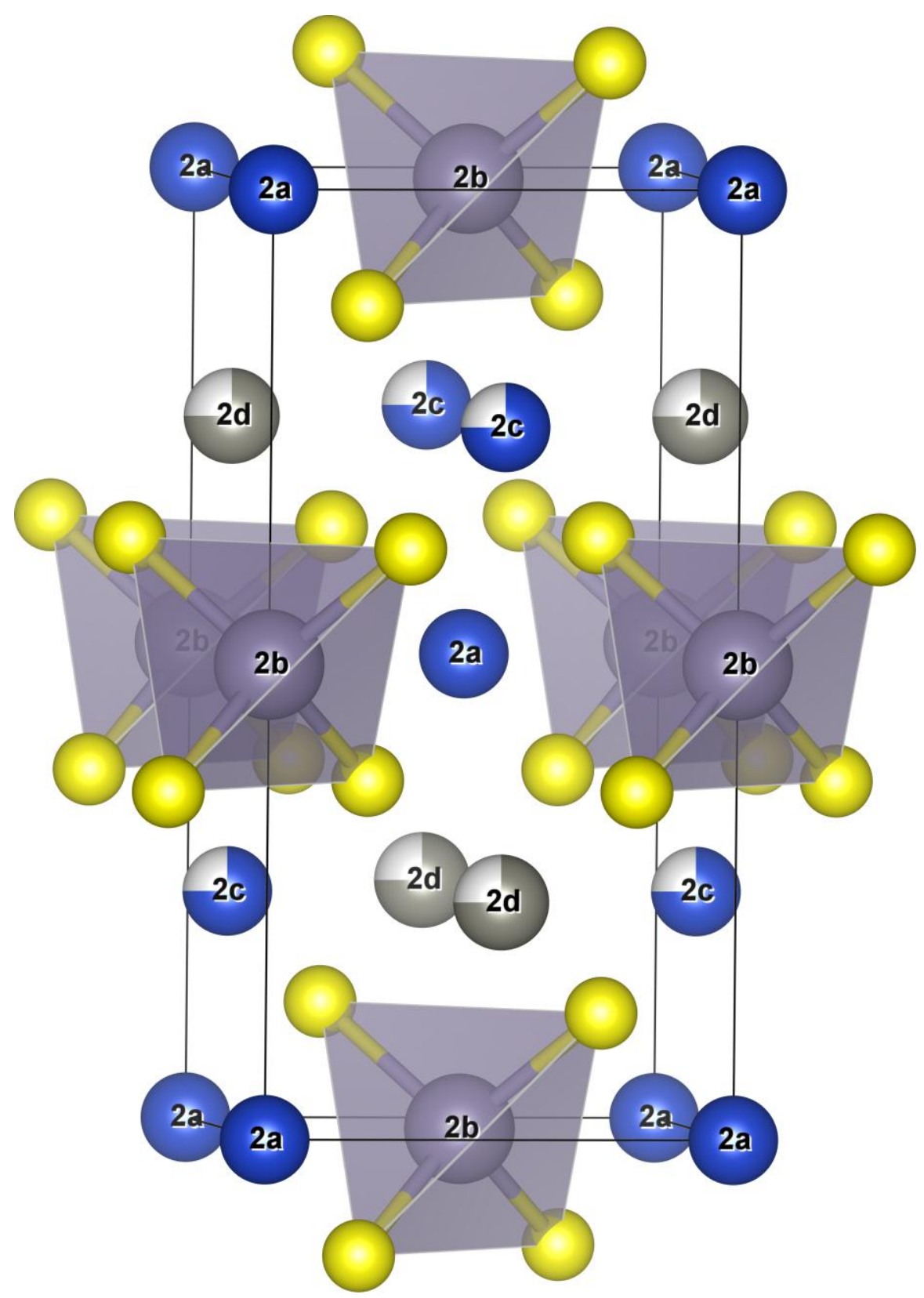

Figure 1. Kesterite type (Hall, Szymanski, \& Stewart, 1978) crystal structure (space group $I \overline{4}$ ). In comparison to the cubic sphalerite type structure, the $c$-axis is doubled due to cation ordering. Anion coordination tetrahedra are shown for the $2 b$ site only, but are very similar for the other sites. In stoichiometric CZTSSe compounds, $2 b$ is occupied by tin, $2 a$ by copper, $2 c$ also preferably by copper, and $2 d$ preferably by zinc (Schorr, 2011). 


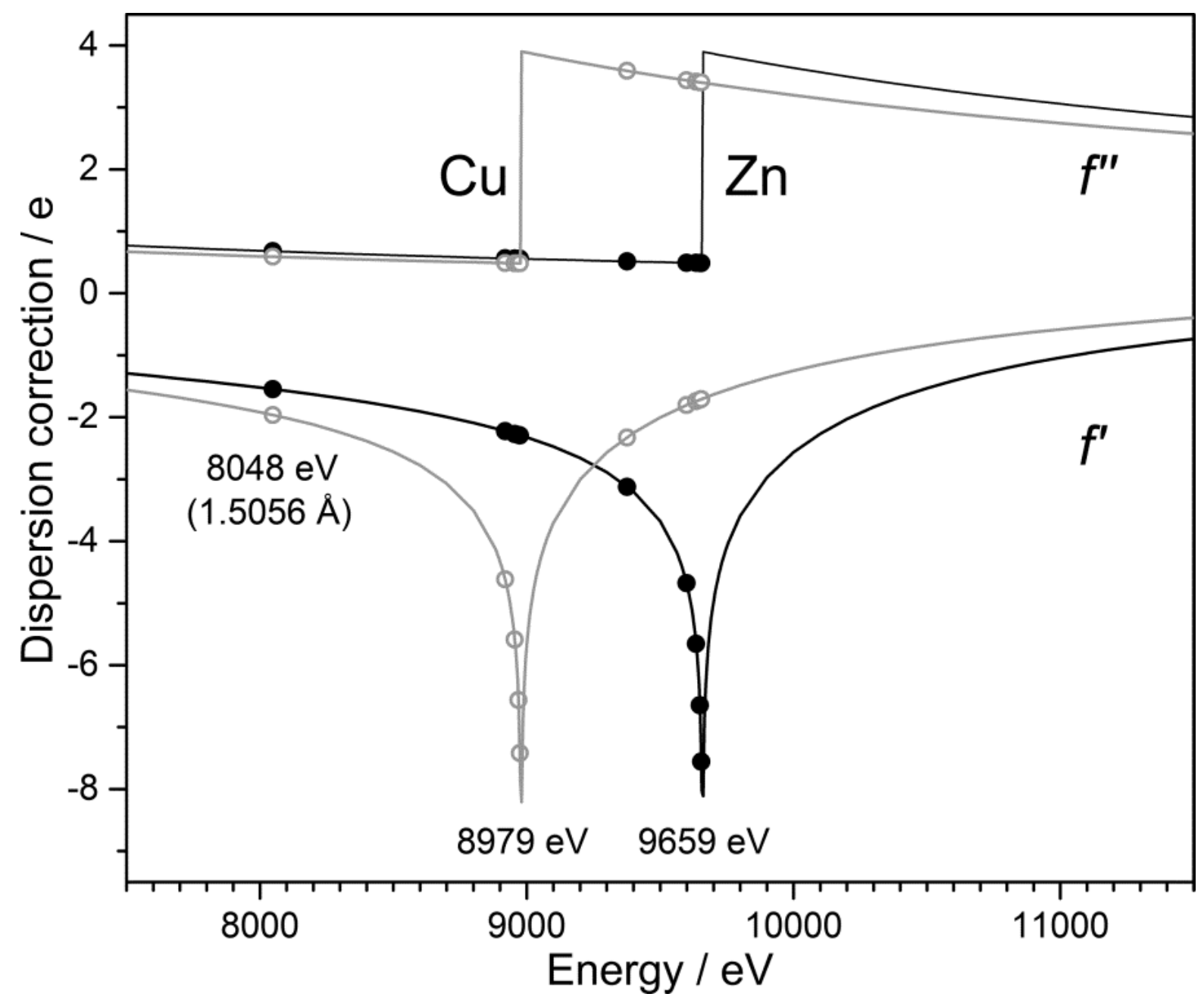

Figure 2. Anomalous X-ray scattering factors $f^{\prime}$ and $f^{\prime \prime}$ for copper (grey) and zinc (black) in the vicinity of respective K-edge (Cromer and Liberman). Dots indicate energies listed in table I. 


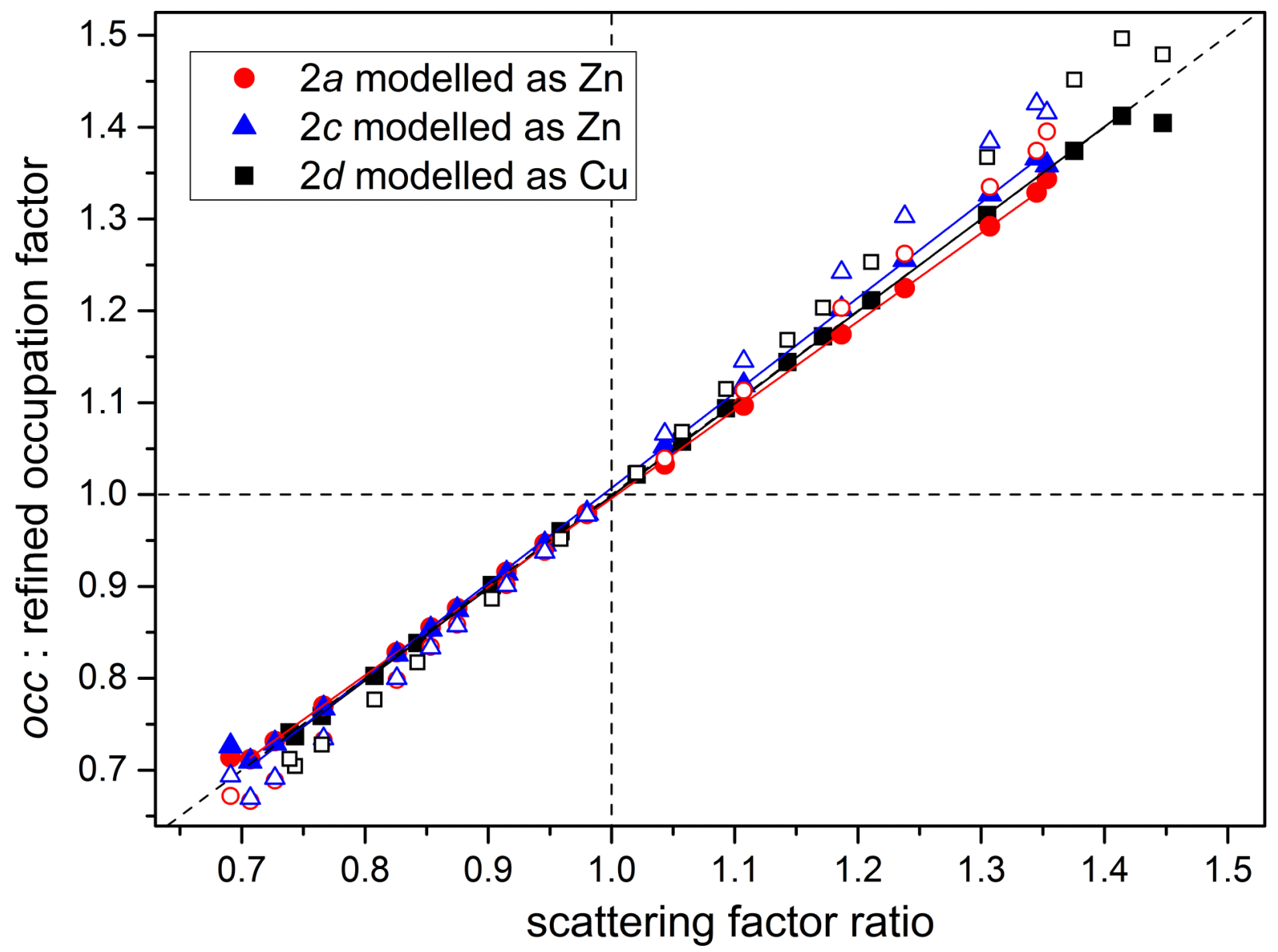

Figure 3. Refined occupation factors for a fully ordered kesterite with exchanged site-occupying species simulated with realistic profile parameters plotted against adjusted scattering factor ratio (full symbols). Ideal limit simulations yield a different slope (open symbols) when plotted against the same ratios. Extreme points deviating from linearity are affected by the $f^{\prime \prime}$ jump at the absorption edge; these can be avoided in experiment. 


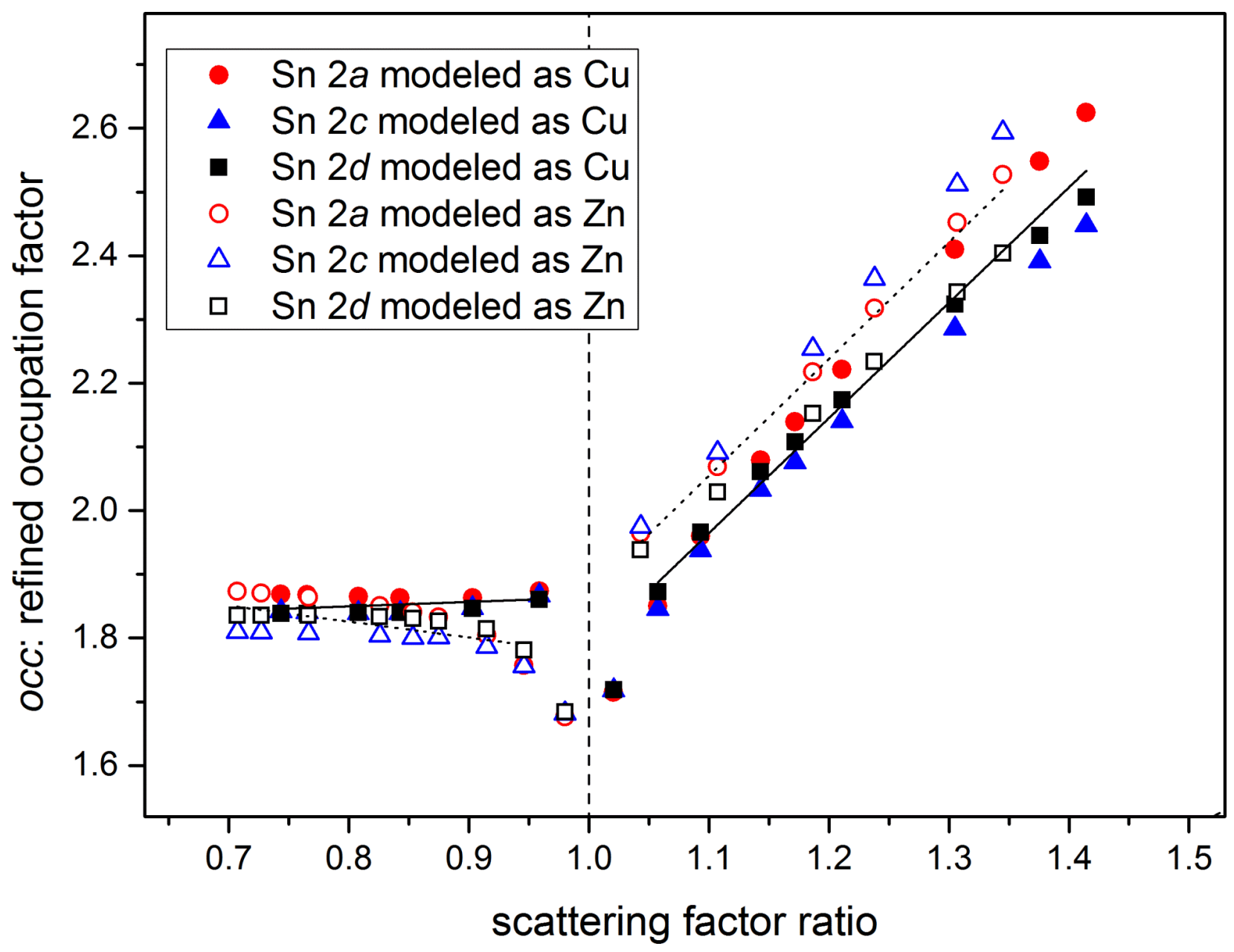

Figure 4. Refined occupation factors for a simulated fully ordered kesterite. The site indicated in the legend was fully occupied by pure tin (Sn) in the calculation of the pattern, but assumed as $\mathrm{Cu}$ or $\mathrm{Zn}$, respectively, in the analysis. Note relatively high deviations from ideal behavior, which is discussed in the text. 


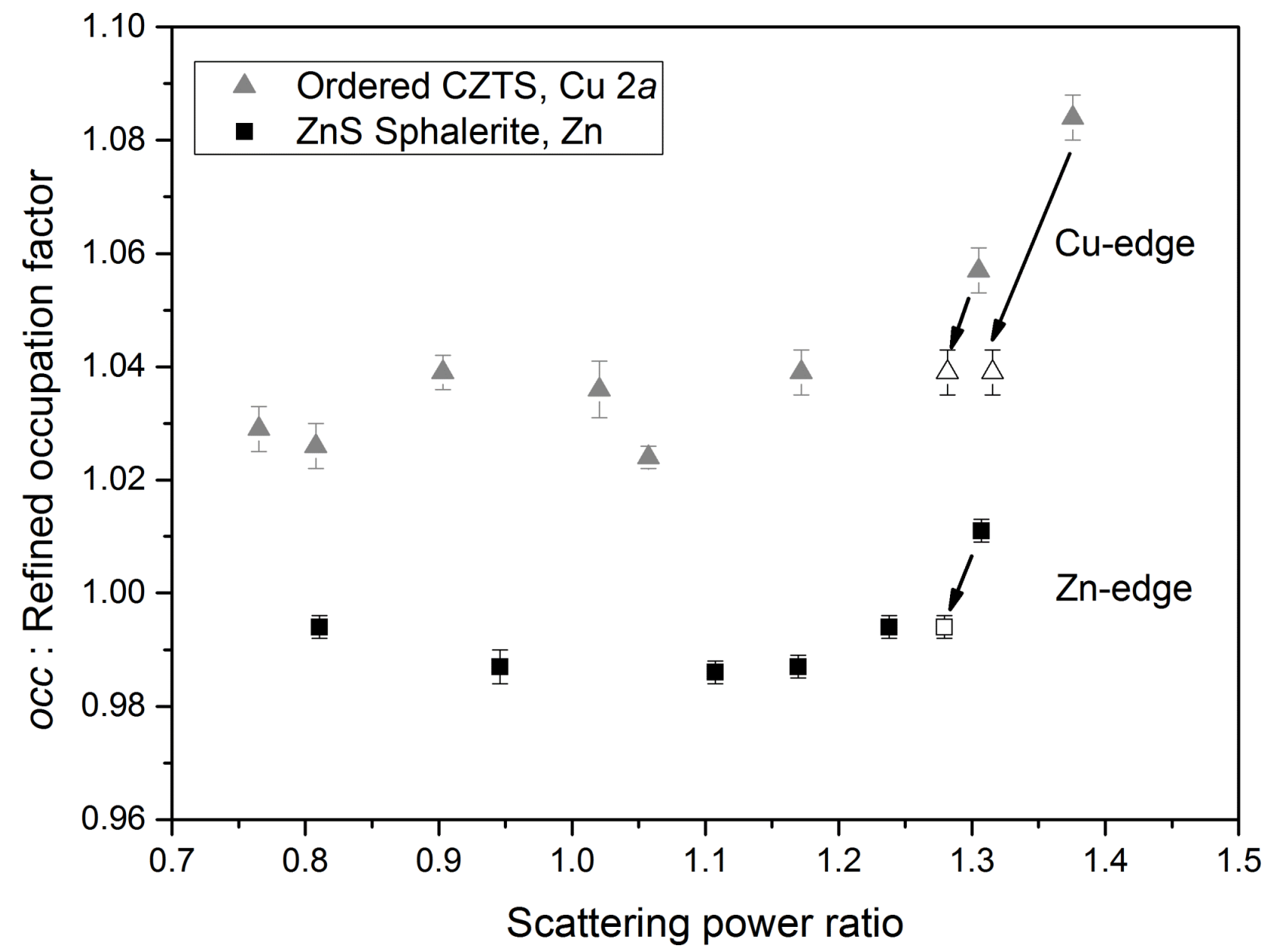

Figure 5. Experimental refined occupation factors of atomic site fully occupied by either copper or zinc only. For energies close to the absorption edge, $f^{\prime}$ had to be adjusted so that always the same occupation factor results. This is independent of systematic errors that can affect the absolute value of the total occupation factor. 


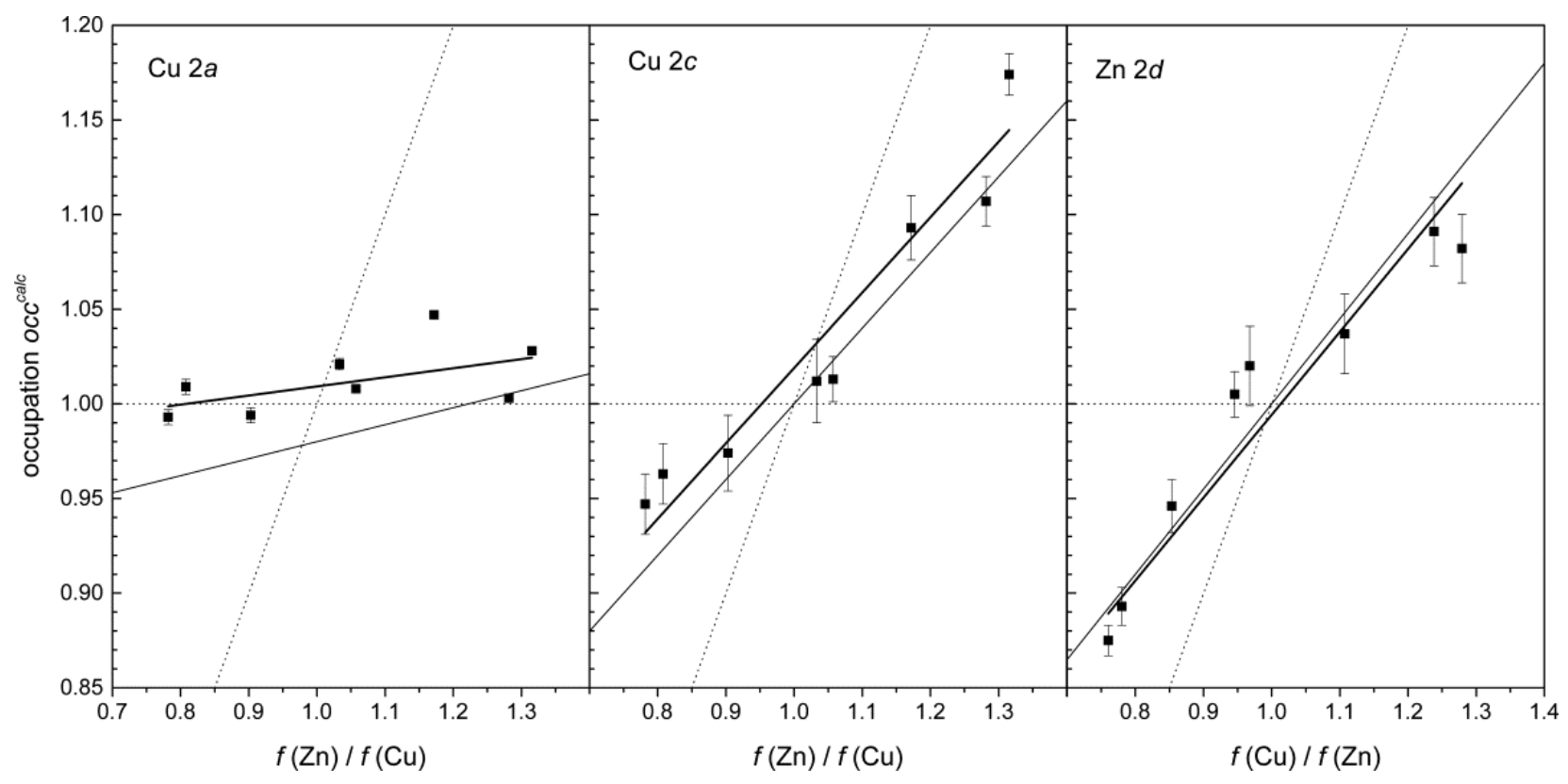

Figure 6. Results of an exemplary analysis with fitted linear dependency (bold solid line). Shown for clarity are the expected line from neutron analysis (thin solid line) and the lines with slope 0 and 1 representing full occupation with Cu or Zn (dotted lines). 